Article

\title{
Glutathione Injection Alleviates the Fluctuation of Metabolic Response under Thermal Stress in Olive Flounder, Paralichthys olivaceus
}

\author{
Seonghye Kim ${ }^{1}{ }^{(D}$, Ahran Kim ${ }^{1,2}$, Seohee Ma ${ }^{1}$, Wonho Lee ${ }^{1}$, Sujin Lee ${ }^{1}$, Dahye Yoon ${ }^{1,3}{ }^{\mathbb{D}}$, \\ Do-Hyung Kim ${ }^{2}$ and Suhkmann Kim ${ }^{1, *}$ \\ 1 Department of Chemistry, Center for Proteome Biophysics, and Chemistry Institute for Functional Materials, \\ Pusan National University, Busan 46241, Korea; seonghyeee@pusan.ac.kr (S.K.); ahran110@naver.com (A.K.); \\ metabomsh@pusan.ac.kr (S.M.); wonholee@pusan.ac.kr (W.L.); isujin@pusan.ac.kr (S.L.); \\ dahyeyoon@korea.kr (D.Y.) \\ 2 Department of Aquatic Life Medicine, Pukyong National University, Busan 48513, Korea; dhkim@pknu.ac.kr \\ 3 Department of Herbal Crop Research, National Institute of Horticultural and Herbal Science, RDA, \\ Eumseong 27709, Korea \\ * Correspondence: suhkmann@pusan.ac.kr; Tel.: +82-51-510-2240
}

Received: 11 November 2019; Accepted: 14 December 2019; Published: 18 December 2019

\begin{abstract}
Continuous increases in water temperature disturb homeostasis and increase oxidative stress in fish. Glutathione (GSH) is an intracellular antioxidant that helps to relieve stress in animals. In this study, we observed the effect of GSH on olive flounder exposed to high temperature using serum parameters and NMR-based metabolomics. Based on the results from the first experiment, $20 \mathrm{mg}$ of GSH was chosen as an effective dose with lower infection rates and mortality. Then, fish were divided into Control, Temp (PS injection), and GSH (glutathione injection) groups, and fish in Temp and GSH groups were exposed to temperature fluctuations $\left(20^{\circ} \mathrm{C} \rightarrow 24^{\circ} \mathrm{C} \rightarrow 27^{\circ} \mathrm{C}\right)$. In OPLS-DA score plots, Temp group was clearly distinguished from the other groups in the kidney. In the liver, the metabolic patterns of GSH group were close to the Temp group on day 4 and became similar to Control group from day 7. Serum parameters did not change significantly, but the deviation in Temp group was greater than that in GSH group. Metabolite levels that were significantly altered included GSH, lactate, O-phosphocholine, and betaine in the kidney and taurine, glucose, and several amino acids in the liver, which were related to antioxidant activity and energy system. Therefore, GSH supplements could relieve thermal stress influencing metabolic mechanisms in fish.
\end{abstract}

Keywords: glutathione injection; thermal stress; metabolomics; NMR; olive flounder

\section{Introduction}

Environmental stressors are detrimental to fish when they are present at concentrations above the normal range. A stressed fish undergoes physiological and biochemical changes to maintain homeostasis and attempts to return to prestress conditions to overcome the stress [1]. Fish are poikilothermic (ectothermic) animals and cannot avoid alternations, such as changes in salinity and temperature and exposure to pollution, in their surrounding environment [2]. Water temperature is important to the well-being and survival of fish [3]. Global climate change is rapidly worsening and one ongoing effect is an increase in the ocean temperatures. According to the Ministry of Oceans and Fisheries, the average water temperature of Korea's coast has risen by $2.89^{\circ} \mathrm{C}$ over the past decade, and the coastal water temperature in July 2018 reached over $28.5^{\circ} \mathrm{C}$ on Jeju Island, which has a lot of aquaculture farms of olive flounder. Olive flounder (Paralichthys olivaceus), one of the major fish species in the aquaculture industry of South Korea, is a stenothermal fish that survives at an 
optimal temperature of $20^{\circ} \mathrm{C}$. In fish, a $10^{\circ} \mathrm{C}$ increase in water temperature will double the rate of physiological function and significantly increase mortality [4]. Most studies have conducted thermal stress experiments at $30-34{ }^{\circ} \mathrm{C}$, which are excessively high temperatures and cannot reflect the effects in the real environment [5-7].

Changes in water temperature beyond the optimal range affect the functioning of fish. Such changes lead to the secretion of cortisol, a primary stress hormone, and to fluctuations in energy metabolism and osmoregulation because of temperature-influenced enzyme traits [8]. In addition, high water temperature causes increases in the generation of reactive oxygen species (ROS) in fish. The overproduction of ROS can damage cellular components, such as cell membranes, DNA, and protein, and can induce oxidative stress [9-11]. Oxidative stress can cause severe consequences from the modification of cellular functions to death. To alleviate oxidative stress, cells possess antioxidant components, including glutathione (glutamyl-cysteinyl-glycine; GSH). GSH works mainly as an antioxidant defense mechanism and is involved in nutrient metabolism, protein synthesis, and the regulation of other cellular events in human and animals [12]. It was confirmed that the inoculation of the vaccine with GSH was effective for the reduction of stress and inflammatory response in calves [13]. And, the effect of GSH in the antioxidant system has been investigated in environmental and chemically stressed fish [14], and the influence of increased temperature on the levels of GSH in multiple tissues has been reported [15]. GSH is mainly given through oral administration to fish in aquaculture farms, but the injection can be a more effective way to administer GSH, because this requires a smaller amount of materials in one treatment, and the duration is longer than the oral route. The injection of GSH into rainbow trout (Oncorhynchus mykiss) rapidly increased GSH levels in the liver, kidney, and gills without the inhibition of additional GSH synthesis, unlike the case in mammalian tissues, and it could serve as an antioxidant $[16,17]$. The GSH system in fish is flexible and can rapidly react to high GSH demands under stressful conditions.

In general, the monitoring of hematological parameters such as plasma cortisol and metabolic enzyme activities are used to detect stress in fish [18-20]. However, these detectable physiological responses cannot always be elicited as stress responses, and the lack of these factors does not always mean that the fish are stressed. Also, they must be complemented with other measurements due to their high variability and they are related to metabolic changes as an adaptation or acclimation mechanism [21]. In previous studies, metabolomics analysis identified a decrease of amino acids including glutamine, tyrosine, and phenylalanine) and alternation in lipid metabolism in high thermal-stressed Atlantic salmon (Salmo salar) [18]. Further, the disturbance of the energy metabolism, including decreased ATP, phosphocreatine, and glycogen levels, has been observed in rainbow trout (O. mykiss) under high temperatures [22]. Using blood parameters, metabolomics can better explain the physiological changes in stressed fish [23]. The metabolomics approach using nuclear magnetic resonance (NMR) enables the determination of the levels of small metabolites with high reproducibility and fast screening along with the evaluation of the ongoing metabolic alteration. The metabolites in intact tissues can be measured using high-resolution magic angle spinning (HR-MAS) NMR without any extraction process of the metabolites and the possibility of experimental loss. In the present study, the appropriate concentration of GSH injection for P. olivaceus was determined. The changes of metabolites in the kidney and liver of P. olivaceus was investigated, and the physiological responses under thermal-stress were confirmed with changes in the hematological parameters and NMR-based metabolomics. The objectives of this study are to elucidate the effect of GSH on stressed fish during an increase in temperature as well as when the fish were maintained at a high temperature.

\section{Results}

\subsection{Glutathione Injection Test}

In the first experiment, natural infection by Edwardsiella piscicida was observed from the first week and over a $40 \%$ infection rate was observed until the third week in the Control and $10 \mathrm{mg}$ GSH groups. 
The $20 \mathrm{mg}$ GSH group showed the lowest infection rate for the experimental period (Table 1). The Control showed the lowest mortality of $8.33 \%$, but the highest infection rate of $60 \%$ at three weeks. This indicates that although fish did not lead to mortality during the observation period, they could cause a potential mortality because they retained bacteria. And, the final mortality rate was $8.33 \%$, $11.83 \%$, and $28.50 \%$ in the Control, $20 \mathrm{mg} \mathrm{GSH}$, and $10 \mathrm{mg}$ GSH groups, respectively, indicating that the injection of $20 \mathrm{mg}$ of GSH was more effective than that of $10 \mathrm{mg}$ of GSH (Figure 1a). The concentration of GSH in liver significantly increased in the injection group compared to the Control group and was maintained for three weeks (Figure 1b). Based on these results, the second experiment was performed using an injection of $20 \mathrm{mg}$ of GSH.

Table 1. Infection rate of olive flounder in the glutathione injection test.

\begin{tabular}{cccc}
\hline Week & Control (\%) & 20 $\mathbf{~ m g ~ G S H ~ ( \% ) ~}$ & $\mathbf{1 0 ~} \mathbf{~ m g ~ G S H ~ ( \% ) ~}$ \\
\hline 0 & & $0 / 5(0)$ & \\
1 & $1 / 5(20)$ & $1 / 5(20)$ & $2 / 5(40)$ \\
3 & $3 / 5(60)$ & $0 / 5(0)$ & $2 / 5(40)$ \\
\hline \multicolumn{4}{c}{$\%$ Percentage of infection. }
\end{tabular}

(a)

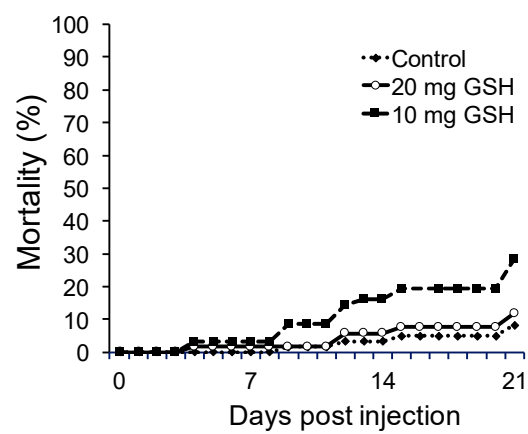

(b)

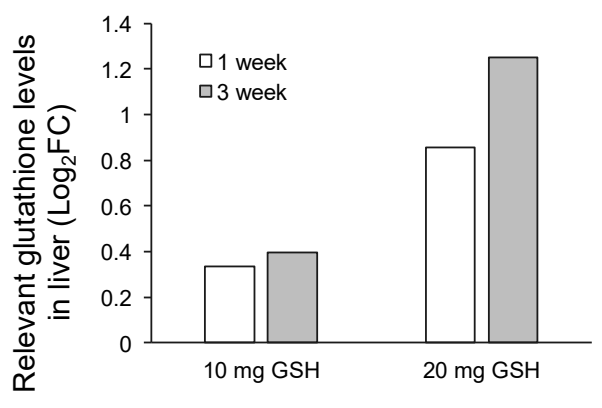

Figure 1. Mortality rate (a) of the glutathione injection experiment and (b) concentrations of glutathione in the liver (a) PS injection (Control), $10 \mathrm{mg}$ of GSH injection (10 mg GSH), and $20 \mathrm{mg}$ of GSH injection (20 mg GSH). (b) The relevant GSH levels ( $\log _{2}$ (fold change)) in the $10 \mathrm{mg}$ and $20 \mathrm{mg} \mathrm{GSH}$ groups compared to the Control group at 0 week were 0.35 and 0.85 at 1 week, and 0.40 and 1.25 at 3 weeks, respectively.

\subsection{Effect of Glutathione on Thermal Stress}

No mortality and infection occurred during the experiment. The variable of this experiment was only a temperature increase, similar to that seen in the environment

\subsubsection{Results of Serum Parameters}

The differences in serum biochemical parameters among the groups are shown in Figure S1. The blood urea nitrogen (BUN), total protein (TP), and total cholesterol (TCHO) were significantly changed $(p<0.05)$ in the Temp group at day 10. Alanine aminotransferase (ALT) and aspartate aminotransferase (AST) in the Temp group showed dramatic increases on days 4 and 10, respectively. Compared to the Temp group, the GSH group had a smaller variation in the measured parameters.

\subsubsection{Metabolic Changes in Kidney and Liver under Thermal Stress}

In the present study, metabolite analysis based on NMR was conducted, and a total of 37 metabolites were assigned in both the kidney and liver. The normalized concentrations and fold changes (FC) of all metabolites are shown in Supplementary Tables S1 and S2, respectively. In the kidney, most 
metabolites changed significantly in the Temp group on day 4. GSH, O-phosphocholine (PC), taurine, glucose, and lactate were significantly increased in Temp group (Figure 2a). Further, betaine and myo-inositol were decreased after day 7. In the liver, GSH, taurine, methionine, glucose and amino acids were identified as significant metabolites (Figure 2b). According to the pathway analysis, the results showed that 16 pathways were identified $(p<0.05)$ (Table 2). The most significant pathways were amino acid metabolism including alanine, aspartate and glutamate metabolism; glycine, serine and threonine metabolism and valine, leucine and isoleucine biosynthesis.

Heat maps visualized the metabolic changes of the Temp and GSH groups exposed to water temperature fluctuation (Figures $3 \mathrm{~b}$ and $4 \mathrm{~b}$ ). Each column displays the average metabolite concentration in each group. Similar trends of metabolic changes in the Control and GSH groups were observed in both organs as the temperature increased. In the kidney, similar patterns were observed in the Control and GSH groups, except for on day 10. The pattern of the GSH group was similar to that of the Control group or located in the middle of the two groups. In the liver, the metabolic pattern in the heat maps of GSH and Temp groups differed from that of the Control group on day 4. However, the metabolites of the GSH group changed, similar to what was observed in the Control group on days 14 .

Table 2. Top significant metabolic pathways from the MetaboAnalysis.

\begin{tabular}{|c|c|c|c|c|}
\hline No. & Pathway Name & Hits & Total & $p$-Value \\
\hline & Amino acid metabolism & & & \\
\hline 1 & Alanine, aspartate and glutamate metabolism & 8 & 28 & $6.41 \times 10^{-7}$ \\
\hline 2 & Arginine biosynthesis & 6 & 14 & $1.23 \times 10^{-6}$ \\
\hline 3 & Glycine, serine and threonine metabolism & 7 & 33 & $3.03 \times 10^{-5}$ \\
\hline 4 & Valine, leucine and isoleucine biosynthesis & 8 & 4 & $4.37 \times 10^{-5}$ \\
\hline 5 & Arginine and proline metabolism & 48 & 5 & 0.004448 \\
\hline 6 & Phenylalanine, tyrosine and tryptophan biosynthesis & 4 & 2 & 0.0049789 \\
\hline 7 & Histidine metabolism & 16 & 3 & 0.010452 \\
\hline \multirow[t]{2}{*}{8} & Phenylalanine metabolism & 10 & 2 & 0.043482 \\
\hline & Metabolism of other amino acids & & & \\
\hline 9 & Glutathione metabolism & 28 & 4 & 0.0082529 \\
\hline 10 & D-Glutamine and D-glutamate metabolism & 6 & 2 & 0.011982 \\
\hline \multirow[t]{2}{*}{11} & beta-Alanine metabolism & 21 & 3 & 0.022376 \\
\hline & Carbohydrate metabolism & & & \\
\hline 12 & Glyoxylate and dicarboxylate metabolism & 32 & 8 & $1.98 \times 10^{-6}$ \\
\hline 13 & Citrate cycle (TCA cycle) & 20 & 4 & 0.0023246 \\
\hline 14 & Pyruvate metabolism & 22 & 4 & 0.0033598 \\
\hline \multirow[t]{2}{*}{15} & Galactose metabolism & 27 & 3 & 0.043482 \\
\hline & Lipid metabolism & & & \\
\hline 16 & Glycerophospholipid metabolism & 36 & 4 & 0.019962 \\
\hline
\end{tabular}




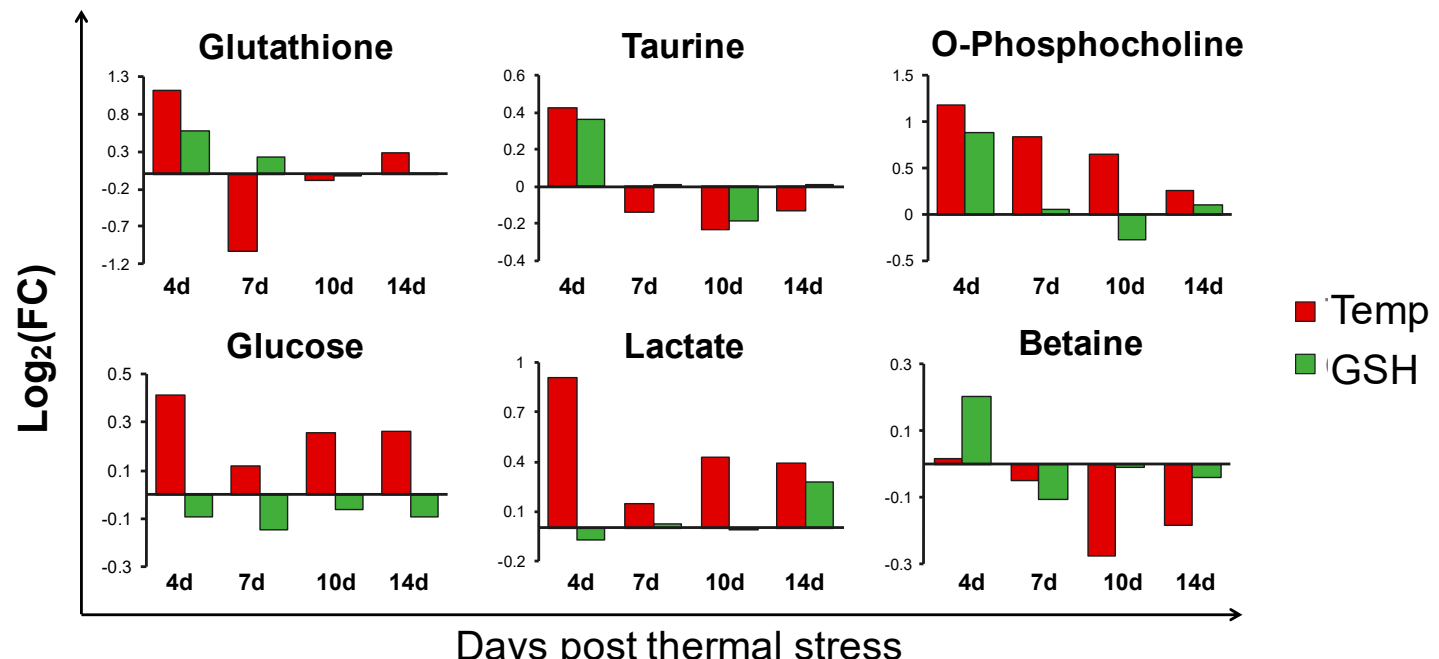

(a)
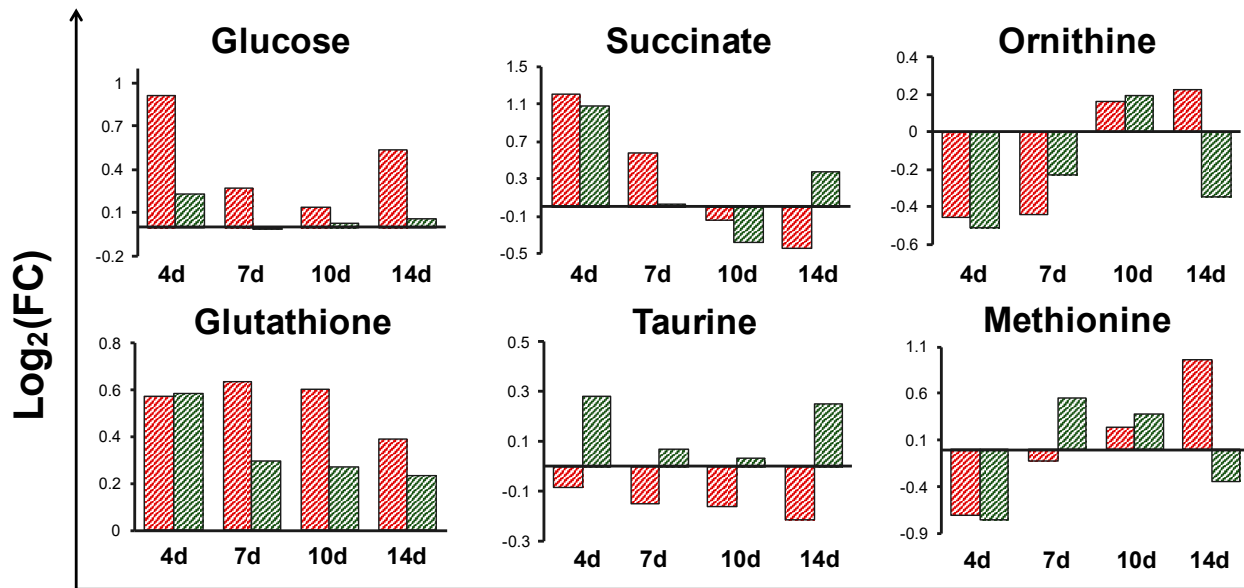

खemp

$4 d \quad 7 d \quad 10 d \quad 14 d$
Methionine

GSH
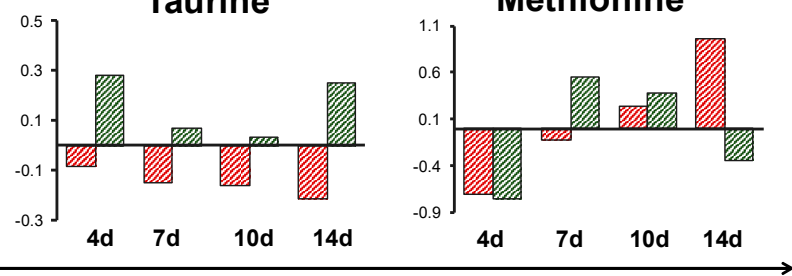

Days post thermal stress

(b)

Figure 2. Relative changes in metabolites of the kidney (a) and liver (b) exposed to thermal stress. Values in Temp and GSH groups are shown as $\log _{2}$ (fold change) relative to the Control group at each sampling time point. d, day; Group (Temp group, Temp; GSH group, GSH); PC, O-Phosphocholine. 


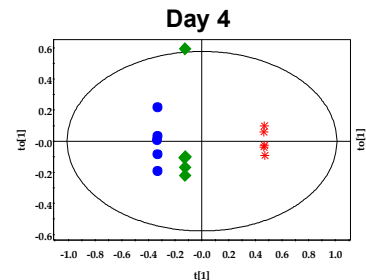

Day 4
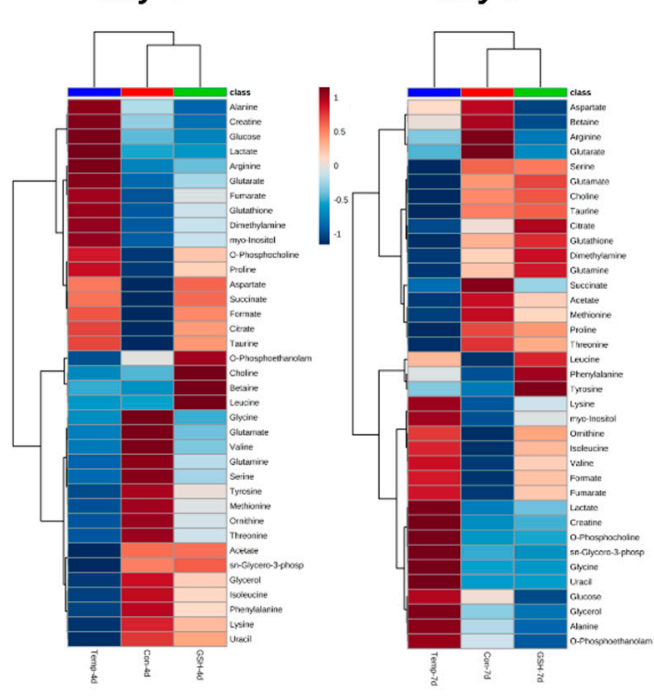

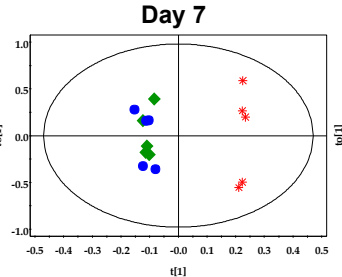

ti11

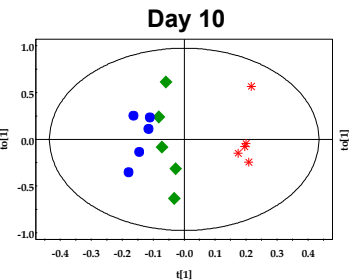

(a)
Day 10

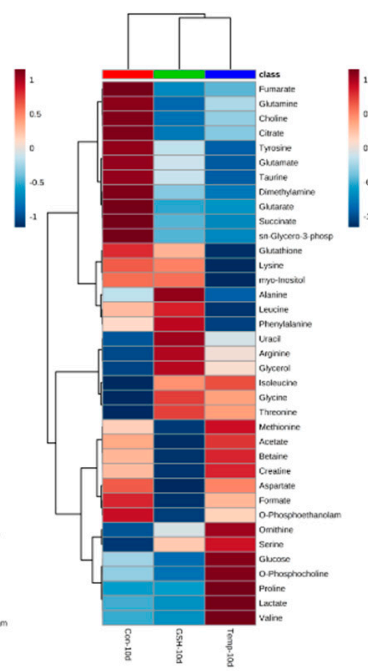

Day 14

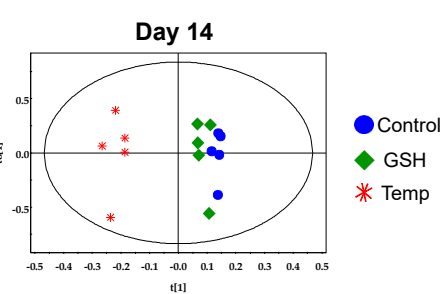

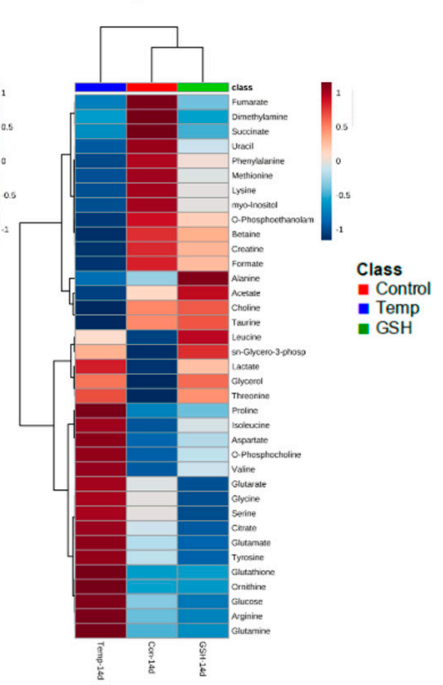

(b)

Figure 3. OPLS-DA score plots (a) and heat maps (b) of the kidney. (a) OPLS-DA score plots of the ${ }^{1} \mathrm{H}$ NMR spectra of the kidney sample for each time point. Dot and color represent sample and group, respectively. Day $4\left(\mathrm{Q}^{2}=0.611\right)$, Day $7\left(\mathrm{Q}^{2}=0.318\right)$, Day $10\left(\mathrm{Q}^{2}=0.191\right)$, and Day $14\left(\mathrm{Q}^{2}=0.135\right)$ ( $n=5$ /group). Red asterisk (Temp group) is separated from the Control and GSH groups. (b) For heat maps, each colored cell corresponds to a concentration value, with groups in rows and metabolites in columns. In the dendrogram, Control and GSH groups are closer than Temp group except for Day 10.

\subsubsection{Results of Multivariate Data Analysis}

Orthogonal partial least squares discriminant analysis (OPLS-DA) score scatter plots of the NMR spectra were conducted to discriminate the metabolic patterns and are shown in Figures 3a and 4a. In the scores plot, each point represents a single spectrum. The results showed different effects of thermal stress depending on the organs. In the kidney, the Temp group were significantly different from the Control and GSH groups with a reasonable $Q^{2}$ value, and the distance between the Control and Temp groups was the largest on day 4 (Figure 3a). The GSH group was clustered with the Control group, whereas the Temp group was on the opposite side until day 14. In the liver, the separation between the Control and the experimental groups was observed on day 4 (Figure 4a). After that, the GSH group showed changes closer to those observed in the Control group. On day 14, the Temp group was significantly discriminated in the score plot. 

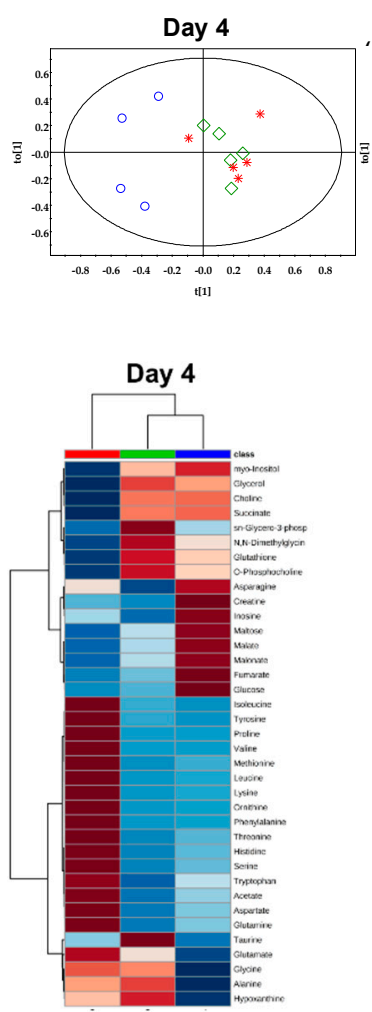
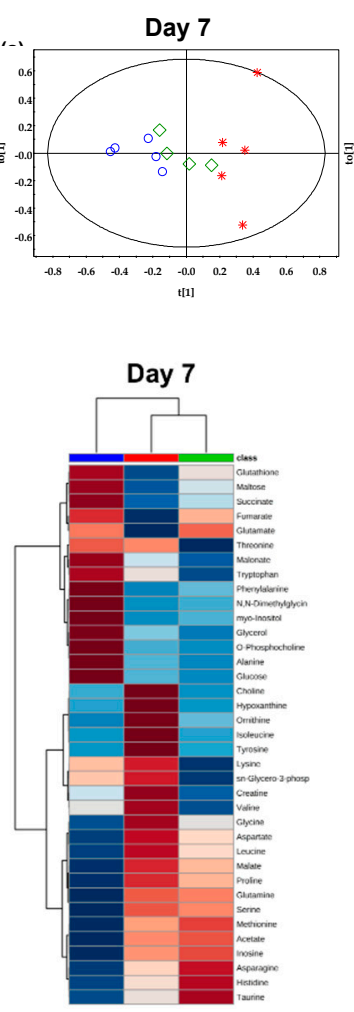

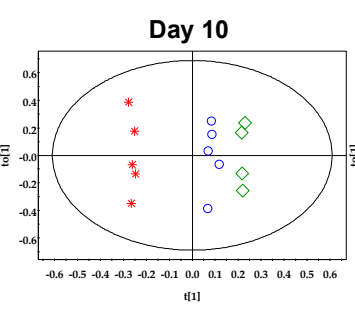

(a)

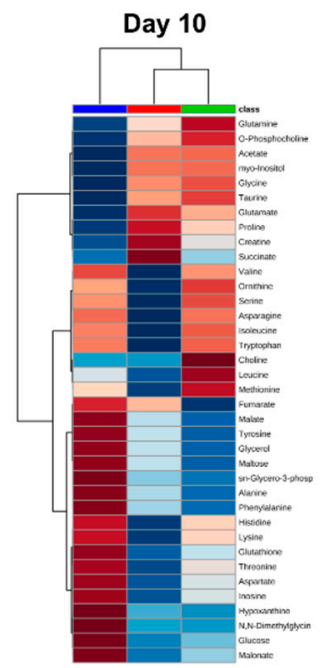

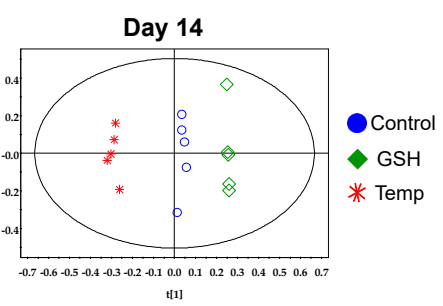

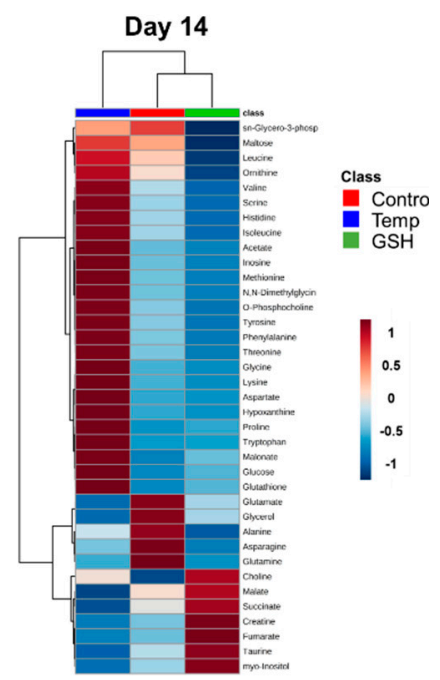

(b)

Figure 4. OPLS-DA score plots (a) and heat maps (b) of the liver. (a) OPLS-DA score plots of the ${ }^{1} \mathrm{H}$ NMR spectra of the liver sample for each time point. Dot and color represent sample and group, respectively. At days 4, 7, and 10, one sample was removed as the outlier; Day $4\left(Q^{2}=0.209\right)$, Day 7 $\left(\mathrm{Q}^{2}=0.531\right)$, Day $10\left(\mathrm{Q}^{2}=0.295\right)$, and Day $14\left(\mathrm{Q}^{2}=0.534, n=5\right)$. The green diamond (GSH group) are closer to the Control group except for day 4. (b) For heat maps, each colored cell corresponds to a concentration value, with groups in rows and metabolites in columns. In the dendrogram, Control, and GSH groups are closer, except for on day 4.

\section{Discussion}

The injection of $20 \mathrm{mg}$ of GSH increased the concentration of GSH in the liver and was effective for survival and defense against the infection. Therefore, exogenous GSH can remain functional in fish tissues. This may affect immune responses via the modulation of T-helper cell, IL-2, antibody production, and expression of immune-related genes [24]. Our results also showed better survival rates and resistance to natural infection with bacteria in a dose-dependent manner.

The activities of AST, ALT, and alkaline phosphatase (ALP) were used as indicators of liver damage in fish [19]. The emission of ALT and AST may be caused by liver damage under high temperatures and they increased in Temp group. Similar results were found in the blunt snout bream (Megalobrama amblycephala) with up-regulation of apoptosis-related gene expression in the liver at $35^{\circ} \mathrm{C}$ [25]. The results obtained for the blood parameters fluctuated because of the high temperature and the deviation in the Temp group was greater than that in the GSH group. Therefore, GSH might help reduce stress-induced fluctuation of biochemical parameters in fish serum by thermal changes.

From the multivariate analysis results, it was determined that the thermal stress affected the variation of the metabolites in the kidney. In the OPLS-DA score plot, the Temp group was always separated from the GSH and Control groups. The well-defined clusters in the heat maps show that the metabolites of the Temp group were altered in an opposite manner to those of the Control and GSH groups. Additionally, the number of significant metabolites with FC $<0.5$ or FC $>1.5$ in the Temp group was the highest on day 4 (Table S1). Therefore, GSH injection reduced the fluctuations caused by high temperatures in the kidney from the first heating stage. On day 4, the GSH level in the Temp 
group was greatly increased along with taurine, in which FC was over 2.0 and decreased expect on days 7 and 10 (Figure 2a). However, in the GSH group, they maintained levels similar to the Control group. These metabolites function as intercellular antioxidants that protect the tissue from oxidative stress. In a previous study [16], GSH level in the kidney was similar to, or more than, that in the liver and it was reported that the GSH injection increased GSH levels the most in the kidney, followed by the liver. This suggests that the kidney of fish is involved in antioxidant protection, similar to the liver. An increase in water temperature led to intercellular metabolic activation producing ROS [16]. GSH in the kidney of the spotted snakehead (Channa punctata) decreased at $32{ }^{\circ} \mathrm{C}$ and it was maintained at a higher level after transfer to $20^{\circ} \mathrm{C}$ [26]. However, in the present study, GSH significantly and continuously decreased due to the maintenance of a high temperature. Together with GSH, taurine is a major intracellular antioxidant and regulates the inflammatory response [27,28]. It has been reported that dietary taurine supplementation can reduce oxidative stress and the disturbance of $\mathrm{Ca}^{2+}$ homeostasis induced by thermal stress in pufferfish (Takifugu obscurus) [29]. After day 4, taurine continuously decreased in the Temp group. It also decreased in the GSH group on day 10, but the GSH levels recovered on day 14 similarly to the Control group. Therefore, heat stress induced decreases in GSH and taurine, and it may be the result of a disorder in the antioxidant defense system of the kidney.

Despite the high glucose levels, the lactate levels in the Temp group also increased significantly on days 4 and 14 (Figure 2a). In the GSH group, the lactate level did not change compared to the Control group, except on day 14. Exposure to acute heat stress can induce the reduction of oxygen in organisms [30]. Lactate is the main product of anaerobic metabolism. The levels of lactate typically increase in stressed fish and lactate was reported to be a potential indicator of heat resistance in the olive flounder [31]. In addition, lactate production has been known to increase to satisfy the energy requirements in turbot (Scophthalmus maximus) [31], sockeye salmon (O. nerka) [32], and killifish (Fundulus heteroclitus) [8] after thermal stress. The results in the present study indicate that the energy metabolism in the kidney was influenced at higher temperatures and the GSH group was less affected.

Additionally, PC and sn-Glycero-3-phosphocholine (GPC) in glycerophospholipid metabolism are significantly changed in kidney. The levels of PC continuously increased in the Temp group, and GPC increased on days 7 and 14 with an FC of over 1.2. PC and GPC are major components of membrane lipids. The increase in PC under stressful conditions may be associated with a collapse of phosphatidylcholine, which is a structural element in the cell membrane [33,34]. It is possible that thermal-induced oxidative stress caused cell disruption and resulted in the release of these metabolites. Besides, the kidney is the organ that controls the osmoregulatory function in teleost fish. The hormone hypersecretion induced by high temperatures can cause mineral metabolic changes, which can affect osmoregulation in fish [35]. After day 10, betaine and myo-inositol significantly decreased in the Temp group (Figure 2a). Although not significant, an increase of betaine in the GSH group was observed on day 4. Betaine is an effective osmoprotectant and functions by accumulating in cells. In addition, it exerts many effects as a methyl group donor for protein synthesis and other metabolically vital substances [36]. Myo-inositol levels and the activation of enzymes related to betaine synthesis were increased to maintain osmolality allostasis in tilapia when exposed to salinity stress [37,38]. Therefore, the decrease in these metabolites likely means that high water temperature leads to the impediment of osmosis mechanisms in the kidney. This result is similar to the osmotic and ionic disturbances reported in handling stressed juvenile Senegalese sole (Solea senegalensis) [39].

Unlike in the kidney, the liver was markedly affected on day 4 by thermal stress and GSH injection can alleviate the effect of thermal stress after the second heating stage in the liver. The liver is the main organ that regulates energy consumption. Energy and oxygen consumption are increased to account for the cost of the stress response under thermal stress exposure [40]. In addition, the stress-induced antioxidant defense and homeostasis disturbance are high-energy-demanding processes. Cortisol is a corticosteroid and is secreted as a primary response to thermal stress in rock goby (Gobius paganellus) [5], coho salmon (O. kisutch) [41], and seabass (Dicentrarchus labrax) [42]. Cortisol stimulates energy metabolism and the breakdown of glycogen to glucose in the liver [43]. In the present study, glucose 
significantly increased in the Temp group, except on day 10, and there was no marked change in the GSH group (Figure 2b). A previous study showed similar results, where an increase in glucose was observed in the liver of the Patagonian blenny (Eleginops maclovinus) at $14^{\circ} \mathrm{C}$, which is $4{ }^{\circ} \mathrm{C}$ higher than the optimal temperature, with decreased glycogen and increased activity of enzymes related to carbohydrate metabolism [8]. On day 4, glucose and succinate significantly increased with significant decreases in aspartate, histidine, isoleucine, leucine, lysine, proline, tyrosine, phenylalanine, and valine in the Temp and GSH groups (Table S2). When exposed to stress, amino acids are converted into the citric acid cycle substrates for energy production $[44,45]$. In the present study, the decreased tyrosine and phenylalanine could be the result of conversion to fumarate. These decreases of amino acids suggest that the energy demands increased and the supplement for energy production was heightened. In addition, glyoxylate and dicarboxylate metabolism was significant in this study, and this participates in energy metabolism as a bypass to the TCA cycle, and plays an important role in many fungi [46]. Additionally, maltose significantly increased in the Temp and GSH groups, except for on day 14. Maltose could be involved in energy replenishment by its degradation into two molecules of glucose. A similar reestablishment of energy metabolism at high temperatures, as has been reported previously in the Atlantic salmon (Salmo salar) [18]. Glucose also increased on day 14. However, the changes in amino acids were the opposite of those seen on day 4 . This may be the result of protein degradation because of the high temperature [47]. Protein degradation produces ammonia, and the ornithine-urea cycle converts ammonia into urea primarily in the liver [48]. A significant decrease of ornithine on days 4 and 7 was observed in both experimental groups, which could be the result of ammonia detoxification. In the heat maps of days 10 and 14, these amino acids are indicated by a red color in the Temp group relative to the Control and GSH groups which are blue. This suggests that thermal-induced protein damage could be alleviated by GSH supplementation.

Compared to the Control group, GSH increased in both groups during the experiment. The elevated GSH levels in the GSH group could be the result of thermal stress and supplementation, consistent with our results in Figure 1b. However, the GSH levels increased more in the Temp group than in the GSH group even though they were placed under the same stressful conditions. The taurine levels increased in the GSH group but decreased in the Temp group relative to the Control group (Figure 2b). After day 4, the taurine level in the GSH group did not change compared to the Control group, but significant increases were observed on day 14 with decreases in GSH. In addition, GSH and taurine decreased in the Temp group on day 14. This suggests that thermal stress at temperatures higher than $27^{\circ} \mathrm{C}$ may increase oxidative stress and affect liver antioxidant mechanisms. Methionine, glycine, and glutamate decreased in the Temp group but did not significantly change in the GSH group. Methionine is trans-sulfurated to cysteine, which is the main precursor of antioxidants such as GSH and taurine (Figure 5) [49]. The decreased methionine could be used to synthesize GSH or taurine. High temperatures may induce intracellular oxidative stress and stressed fish need to activate GSH synthesis. In the Temp group, methionine was more biased to GSH synthesis than taurine in the Temp group and this lack of taurine continued to day 14. However, in the GSH group, taurine was maintained at a level higher than or similar to that in the Control group and it could support the anti-oxidative effect [35]. This result suggests that thermal stress causes excessive synthesis of GSH in the liver as a defense against oxidative stress and GSH supplement could maintain homeostasis of antioxidants. 


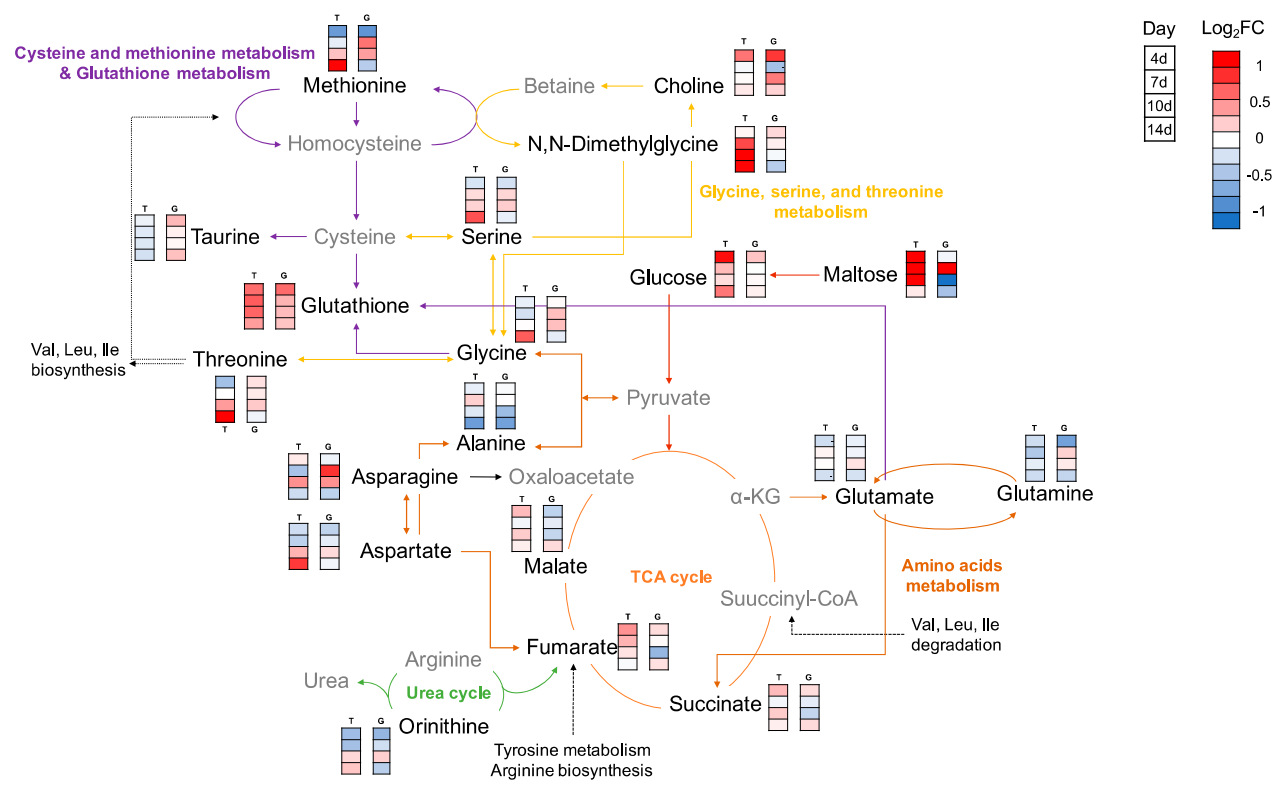

Figure 5. Schematic figure of metabolic pathways in liver. The red and blue colors in the bar show the increase and decrease in relative concentrations of metabolites compared to the Control, respectively. The orders from up to down represent day post thermal stress. GSH increased in both groups as temperature increased. Grey colored metabolites were not assigned in this study. T, Temp group; G, GSH group; d, day; FC, fold change; $\alpha-K G, \alpha$-ketoglutarate.

To the best of our knowledge, this is the first study to evaluate the effects of GSH on thermal-stressed fish using combined analysis with NMR-based metabolomics and serum biochemical tests. The $20 \mathrm{mg}$ GSH injection was effective in lowering the infection rate and mortality. After the fish were exposed to thermal stress, metabolic profiling was conducted and significant changes due to high temperatures and GSH injection were identified by comparison with the Control group. The results showed that high temperature induced dynamic metabolic changes and the stress response to heat was different depending on the organ. The fluctuations in metabolite changes and hematological parameters were smaller in the GSH group than in the Temp group. NMR-based metabolomics is a powerful approach to elucidate metabolic changes as a response to stress and it can detect stress even if the physiological response does not change significantly. These findings confirmed the efficacy of GSH in improving homeostasis and alleviating the effects of heat stress. Because fish are exposed to multiple stressors, further studies are required to validate that GSH is efficacious for various stresses in fish and not only for heat stress as in the present study.

\section{Materials and Methods}

\subsection{Glutathione Injection Test}

All animal experimental procedures were carried out in accordance with the guidelines and regulations and with the ethical approval from the Ethics Committee of Pukyong National University (approval number: 2017-2010).

The first experiment was designed to determine the effective dose and duration of GSH. Healthy olive flounder (average body weight $(\mathrm{BW})=35 \mathrm{~g}$ ) were obtained from a commercial fish farm in South Korea and the fish were acclimatized in a 1000-L flow-through tank at the Institute of Fisheries Science, Pukyong National University before the commencement of experiments under rearing conditions in aerated and circulated seawater at $20^{\circ} \mathrm{C}$. A total of 180 fish were randomly split into six tanks (30 fish in 250-L flow-through tanks). A GSH (reduced glutathione) suspension for inoculation was prepared by dilution in sterile physiological saline (PS). Intramuscular injections were performed with $100 \mu \mathrm{L}$ of PS into the fish in tank 1 (Control), $100 \mu \mathrm{L}$ of $10 \mathrm{mg} / \mathrm{kg}$ BW GSH into the fish in tank 2 (10 mg GSH), and 
$20 \mathrm{mg} / \mathrm{kg}$ BW GSH into the fish in tank 3 with $100 \mu \mathrm{L}$ ( $20 \mathrm{mg}$ GSH). Fish were monitored for 3 weeks and the mortality and infection rate were measured at 0,1 , and 3 weeks. Five fish were sampled from each group to investigate the infection rate and GSH concentration in the liver. GSH concentration was measured using NMR. Bacterial infection was observed by stamping the fish kidney on brain heart infusion agar (Difco) supplemented with $1 \% \mathrm{NaCl}$ and incubated at $28^{\circ} \mathrm{C}$ for $24 \mathrm{~h}$.

\subsection{Thermal Stress Exposure}

For the second experiment, 165 fish with an average BW of $30 \mathrm{~g}$ were obtained and divided into three groups ( $n=55$ in a tank): No change in water temperature after injection with PS (Control group); change in water temperature after injection with PS (Temp group); and change in water temperature after injection with GSH (GSH group). A total of $200 \mu \mathrm{L}$ of PS was injected into the Control and Temp groups and the same volume of $20 \mathrm{mg} / \mathrm{kg}$ BW GSH into the fish of the GSH group. In the Temp and GSH groups, the water temperature was gradually increased by $1{ }^{\circ} \mathrm{C}$ each day for 4 days after injection at $20^{\circ} \mathrm{C}$ (day 4 , first heating). After keeping at a constant $24^{\circ} \mathrm{C}$ for 3 days (day 7 , first maintenance), the temperature was increased to $27^{\circ} \mathrm{C}$ (day 10, second heating) and maintained for 3 days at $27^{\circ} \mathrm{C}$ (day 14, second maintenance) (Figure 6). The Control group was maintained at $20^{\circ} \mathrm{C}$ over the experimental period. The experiment lasted for 2 weeks and the fish were not fed to minimize the stress caused by water contamination. Five fish were sampled randomly from each tank at days 4, 7, 10, and 14, and euthanized using MS-222. Blood was taken from the caudal vein and allowed to stand for 15 min at room temperature $\left(20-25^{\circ} \mathrm{C}\right)$ followed by $4{ }^{\circ} \mathrm{C}$ overnight, and the serum was isolated after centrifugation at $6500 \mathrm{rpm}$ for $5 \mathrm{~min}$ at $4{ }^{\circ} \mathrm{C}$. The kidney and liver samples were removed aseptically, and all samples were stored at $-80^{\circ} \mathrm{C}$ until subsequent use.

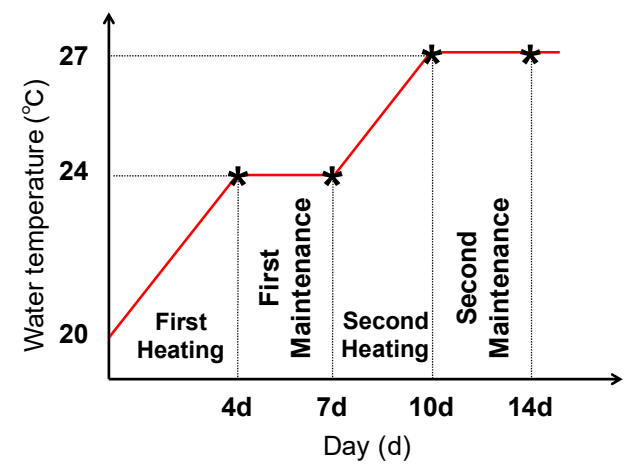

Figure 6. Scheme of the thermal stress experiment. Red line and asterisk represent water temperature changes and sampling time points for experiment, respectively.

\subsection{Analysis of Biochemical Parameters in Serum}

Blood samples were taken from each group at days $0,4,7,10$, and 14. For biochemical analysis in serum, AST, ALT, BUN, TCHO, TP, and ALP were measured using a FUJI DRI-CHEM 4000i (FUJI PHOTO FILM Co., Tokyo, Japan). Statistical analyses were conducted with one-way analysis of variance for multiple comparisons (ANOVA).

\section{4. ${ }^{1}$ H NMR Measurement}

For HR-MAS NMR measurements, $20 \mathrm{mg}$ of an intact kidney sample $(n=5)$ was weighed and carefully placed into a $4 \mathrm{~mm}$ NMR nanotube. A total of $20 \mu \mathrm{L}$ of phosphate buffer ( $\mathrm{pH} 7.4$ ) in deuterated water $\left(\mathrm{D}_{2} \mathrm{O}\right)$ containing $2 \mathrm{mM}$ 3-trimethylsilyl-2,2,3,3-tetradeuteropropionicacid- $\mathrm{d}_{4}$ (TSP- $\mathrm{d}_{4}$, Sigma-Aldrich, St. Louis, MO, USA) was added as an internal chemical shift standard. The liver is inappropriate for HR-MAS because it contains lots of lipids and the peaks of lipids are broad with a wide range. Therefore, polar metabolites were extracted. The lyophilized liver samples were homogenized with a mortar and pestle. The homogenized samples were extracted 
with acetonitrile/water $(1: 1, \mathrm{v} / \mathrm{v})$ [50]. The supernatant was collected after centrifugation (3000 rpm, $4{ }^{\circ} \mathrm{C}, 10 \mathrm{~min}$ ) and freeze-dried. Completely dried samples were reconstituted with $700 \mu \mathrm{L}$ of $\mathrm{D}_{2} \mathrm{O}$ containing $2 \mathrm{mM}$ TSP- $\mathrm{d}_{4}$ and transferred into a $5 \mathrm{~mm}$ NMR tube. ${ }^{1} \mathrm{H}-\mathrm{NMR}$ spectra of the intact kidney tissues were measured using a $600 \mathrm{MHz}$ HR-MAS NMR spectrometer with a nano-NMR probe (Agilent Technologies, Santa Clara, CA, USA). The spinning rate was $2050 \mathrm{~Hz}$ with a degree of magic-angle $\left(54.74^{\circ}\right)$ at a temperature of $24.85^{\circ} \mathrm{C}(298 \mathrm{~K})$. The spectra of the liver extracts were acquired using a liquid probe. A Carr-Purcell-Meiboom-Gill pulse (CPMG) sequence was used to suppress the large peaks of high molecular mass compounds. Spectra were acquired using an acquisition time of 1.703 $\mathrm{s}$, relaxation delay of $1 \mathrm{~s}$, and 128 transients. The acquired spectra were phased, and the baseline was corrected and referenced to the TSP- $\mathrm{d}_{4}$ peak (chemical shift of $0 \mathrm{ppm}$ ) using VnmrJ 4.2 software (Aligent Technologies, Santa Clara, CA, USA).

\subsection{Statistical Analysis of ${ }^{1} \mathrm{H} N M R$ Data}

All spectra were analyzed using the Chenomx NMR Suite 7.1 Professional (Chenomx Inc., Edmonton, AB, Canada) for the identification and quantification of metabolites based on a $600 \mathrm{MHz}$ NMR library database. The concentrations of metabolites were normalized by the sum of total concentration to reduce sample-to-sample variation. The statistical significance of metabolites was determined using a $t$-test (two-tailed, equal variances, $p<0.05$ ). The FC of each metabolite was calculated as being divided by the average of the Control group. Multivariate statistical analysis was performed to confirm the distinction in the metabolic patterns between the Control and thermal-stressed groups. The region of the spectra between 0.5 and $9.0 \mathrm{ppm}$ was binned with a binning size of $0.001 \mathrm{ppm}$. The areas of residual water and sideband were removed. The binned data of each NMR spectrum were normalized with Pareto scaling and imported into the OPLS-DA model using SIMCA P+ (Umetrics, Sweden). The horizontal component of the scattered OPLS-DA score plots indicates the variation between the group. The $\mathrm{Q}^{2}$ parameter was used to evaluate the predictability. For an overview of the metabolites among groups, a heat map of the metabolites concentrations was performed using MetaboAnalyst 4.0. The Kyoto Encyclopedia of Genes and Genomes (KEGG) pathway analysis for the assigned metabolites was performed using, MetaboAnalyst 4.0 with a threshold limit of $p<0.05$.

Supplementary Materials: The following are available online at http://www.mdpi.com/2218-1989/10/1/3/s1, Table S1: Normalized concentration of metabolites in the kidney, Table S2: Normalized concentration of metabolites in the liver, Figure S1: Changes in the biochemical parameters of serum in olive flounder after thermal stress.

Author Contributions: S.K. (Seonghye Kim) and S.L. performed the NMR experiments; A.K. and D.-H.K. performed fish experiments; A.K. analysed the serum biochemical test; S.M., W.L. and D.Y. analysed the experimental data; S.K. (Seonghye Kim) and S.K. (Suhkmann Kim) wrote the paper. All authors have read and agreed to the published version of the manuscript.

Funding: This research was part of a project titled "Omics based on fishery disease control technology development and industrialization (20150242)", funded by the Ministry of Oceans and Fisheries, Republic of Korea and the National Research Foundation of Korea (NRF-2017R1A2B4006648) funded by the Ministry of Education.

Conflicts of Interest: The authors declare no conflicts of interest. The founding sponsors had no role in the design of the study; collection, analyses, or interpretation of the data; writing of the manuscript; or decision to publish the results.

\section{References}

1. Donelson, J.M.; Munday, P.L.; McCormick, M.I.; Pankhurst, N.W.; Pankhurst, P.M. Effects of elevated water temperature and food availability on the reproductive performance of a coral reef fish. Mar. Ecol. Prog. Ser. 2010, 401, 233-243. [CrossRef]

2. Matoo, O.B.; Ivanina, A.V.; Ullstad, C.; Beniash, E.; Sokolova, I.M. Interactive effects of elevated temperature and $\mathrm{CO}_{2}$ levels on metabolism and oxidative stress in two common marine bivalves (Crassostrea virginica and Mercenaria mercenaria). Comp. Biochem. Phys. A 2013, 164, 545-553. [CrossRef] [PubMed] 
3. Logan, C.A.; Buckley, B.A. Transcriptomic responses to environmental temperature in eurythermal and stenothermal fishes. J. Exp. Biol. 2015, 218, 1915-1924. [CrossRef] [PubMed]

4. Di Santo, V.; Bennett, W.A. Effect of rapid temperature change on resting routine metabolic rates of two benthic elasmobranchs. Fish Physiol. Biochem. 2011, 37, 929-934. [CrossRef] [PubMed]

5. Vinagre, C.; Madeira, D.; Mendonca, V.; Dias, M.; Roma, J.; Diniz, M.S. Effect of increasing temperature in the differential activity of oxidative stress biomarkers in various tissues of the Rock goby, Gobius paganellus. Mar. Environ. Res. 2014, 97, 10-14. [CrossRef] [PubMed]

6. Rossi, A.; Bacchetta, C.; Cazenave, J. Effect of thermal stress on metabolic and oxidative stress biomarkers of Hoplosternum littorale (Teleostei, Callichthyidae). Ecol. Indic. 2017, 79, 361-370. [CrossRef]

7. Hu, W.T.; Guo, W.L.; Meng, A.Y.; Sun, Y.; Wang, S.F.; Xie, Z.Y.; Zhou, Y.C.; He, C.Z. A metabolomic investigation into the effects of temperature on Streptococcus agalactiae from Nile tilapia (Oreochromis niloticus) based on UPLC-MS/MS. Vet. Microbiol. 2017, 210, 174-182. [CrossRef]

8. Oyarzun, R.; Munoz, J.L.P.; Pontigo, J.P.; Morera, F.J.; Vargas-Chacoff, L. Effects of acclimation to high environmental temperatures on intermediary metabolism and osmoregulation in the sub-Antarctic notothenioid Eleginops maclovinus. Mar. Biol. 2018, 165. [CrossRef]

9. Lushchak, V.I.; Bagnyukova, T.V. Temperature increase results in oxidative stress in goldfish tissues. 1. Indices of oxidative stress. Comp. Biochem. Phys. C 2006, 143, 30-35. [CrossRef]

10. Almroth, B.C.; Sturve, J.; Berglund, A.; Forlin, L. Oxidative damage in eelpout (Zoarces viviparus), measured as protein carbonyls and TBARS, as biomarkers. Aquat. Toxicol. 2005, 73, 171-180. [CrossRef]

11. Cheng, C.H.; Yang, F.F.; Liao, S.A.; Miao, Y.T.; Ye, C.X.; Wang, A.L.; Tan, J.W.; Chen, X.Y. High temperature induces apoptosis and oxidative stress in pufferfish (Takifugu obscurus) blood cells. J. Therm. Biol. 2015, 53, 172-179. [CrossRef] [PubMed]

12. Kwon, D.H.; Lee, H.; Park, C.; Hong, S.H.; Hong, S.H.; Kim, G.Y.; Cha, H.J.; Kim, S.; Kim, H.S.; Hwang, H.J.; et al. Glutathione Induced Immune-Stimulatory Activity by Promoting M1-Like Macrophages Polarization via Potential ROS Scavenging Capacity. Antioxidants 2019, 8, 413. [CrossRef] [PubMed]

13. Cha, C.N.; Chae, W.S.; Park, E.K.; Yoo, C.Y.; Son, S.E.; Kim, S.; Lee, H.J. Effects of reduced glutathione on stress and inflammatory response in Korean native calves vaccinated with foot-and-mouth disease vaccine. J. Prev. Vet. Med. 2018, 42, 37-40. [CrossRef]

14. Sukhovskaya, I.V.; Borvinskaya, E.V.; Smirnov, L.P.; Kochneva, A.A. Role of Glutathione in Functioning of the System of Antioxidant Protection in Fish (Review). Inland Water Biol. 2017, 10, 97-102. [CrossRef]

15. Leggatt, R.A.; Brauner, C.J.; Schulte, P.M.; Iwama, G.K. Effects of acclimation and incubation temperature on the glutathione antioxidant system in killifish and RTH-149 cells. Comp. Biochem. Phys. A 2007, 146, 317-326. [CrossRef] [PubMed]

16. Leggatt, R.A.; Iwama, G.K. Exogenous glutathione can increase glutathione levels in tissues of rainbow trout (Oncorhynchus mykiss) through extracellular breakdown and intracellular synthesis. Comp. Biochem. Phys. C 2009, 150, 322-328. [CrossRef] [PubMed]

17. Otto, D.M.E.; Sen, C.K.; Hidiroglou, N.; Madere, R.; Moon, T.W. Role of exogenous glutathione in teleost fish and its effects on antioxidant defense responses in rainbow trout exposed to 3, 3', 4, 4' -tetrachlorobiphenyl. Fish Physiol. Biochem. 1997, 16, 449-457. [CrossRef]

18. Kullgren, A.; Jutfelt, F.; Fontanillas, R.; Sundell, K.; Samuelsson, L.; Wiklander, K.; Kling, P.; Koppe, W.; Larsson, D.G.J.; Bjornsson, B.T.; et al. The impact of temperature on the metabolome and endocrine metabolic signals in Atlantic salmon (Salmo salar). Comp. Biochem. Phys. A 2013, 164, 44-53. [CrossRef]

19. Cheng, C.H.; Guo, Z.X.; Luo, S.W.; Wang, A.L. Effects of high temperature on biochemical parameters, oxidative stress, DNA damage and apoptosis of pufferfish (Takifugu obscurus). Ecotoxicol. Environ. Saf. 2018, 150, 190-198. [CrossRef]

20. Wen, B.; Jin, S.R.; Chen, Z.Z.; Gao, J.Z. Physiological responses to cold stress in the gills of discus fish (Symphysodon aequifasciatus) revealed by conventional biochemical assays and GC-TOF-MS metabolomics. Sci. Total Environ. 2018, 640-641, 1372-1381. [CrossRef]

21. Martínez-Porchas, M.; Martínez-Córdova, L.R.; Ramos-Enriquez, R. Cortisol and glucose: Reliable indicators of fish stress? Pan-Am. J. Aquat. Sci. 2009, 4, 158-178.

22. Viant, M.R.; Werner, I.; Rosenblum, E.S.; Gantner, A.S.; Tjeerdema, R.S.; Johnson, M.L. Correlation between heat-shock protein induction and reduced metabolic condition in juvenile steelhead trout (Oncorhynchus mykiss) chronically exposed to elevated temperature. Fish Physiol. Biochem. 2003, 29, 159-171. [CrossRef] 
23. Gandar, A.; Laffaille, P.; Canlet, C.; Tremblay-Franco, M.; Gautier, R.; Perrault, A.; Gress, L.; Mormede, P.; Tapie, N.; Budzinski, H.; et al. Adaptive response under multiple stress exposure in fish: From the molecular to individual level. Chemosphere 2017, 188, 60-72. [CrossRef] [PubMed]

24. Fratelli, M.; Goodwin, L.O.; Orom, U.A.; Lombardi, S.; Tonelli, R.; Mengozzi, M.; Ghezzi, P. Gene expression profiling reveals a signaling role of glutathione in redox regulation. Proc. Natl. Acad. Sci. USA 2005, 102, 13998-14003. [CrossRef]

25. Bing, L.; Sun, S.M.; Jian, Z.; Su, Y.L.; Zhang, W.X.; Ge, X.P. Transcriptome profiling and histology changes in juvenile blunt snout bream (Megalobrama amblycephala) liver tissue in response to acute thermal stress. Genomics 2019, 111, 242-250. [CrossRef]

26. Kaur, M.; Atif, F.; Ali, M.; Rehman, H.; Raisuddin, S. Heat stress-induced alterations of antioxidants in the freshwater fish Channa punctata Bloch. J. Fish Biol. 2005, 67, 1653-1665. [CrossRef]

27. Atmaca, G. Antioxidant effects of sulfur-containing amino acids. Yonsei Med. J. 2004, 45, 776-788. [CrossRef]

28. Elnesr, S.S.; Elwan, H.A.M.; Xu, Q.Q.; Xie, C.; Dong, X.Y.; Zou, X.T. Effects of in ovo injection of sulfur-containing amino acids on heat shock protein 70, corticosterone hormone, antioxidant indices, and lipid profile of newly hatched broiler chicks exposed to heat stress during incubation. Poult. Sci. 2019, 98, 2290-2298. [CrossRef]

29. Cheng, C.H.; Guo, Z.X.; Wang, A.L. The protective effects of taurine on oxidative stress, cytoplasmic free-Ca2+ and apoptosis of pufferfish (Takifugu obscurus) under low temperature stress. Fish Shellfish Immunol. 2018, 77, 457-464. [CrossRef]

30. Currie, S.; Ahmady, E.; Watters, M.A.; Perry, S.F.; Gilmour, K.M. Fish in hot water: Hypoxaemia does not trigger catecholamine mobilization during heat shock in rainbow trout (Oncorhynchus mykiss). Comp. Biochem. Physiol. Part A Mol. Integr. Physiol. 2013, 165, 281-287. [CrossRef]

31. Lu, Y.L.; Wu, Z.H.; Song, Z.C.; Xiao, P.; Liu, Y.; Zhang, P.J.; You, F. Insight into the heat resistance of fish via blood: Effects of heat stress on metabolism, oxidative stress and antioxidant response of olive flounder Paralichthys olivaceus and turbot Scophthalmus maximus. Fish Shellfish Immun. 2016, 58, 125-135. [CrossRef] [PubMed]

32. Steinhausen, M.F.; Sandblom, E.; Eliason, E.J.; Verhille, C.; Farrell, A.P. The effect of acute temperature increases on the cardiorespiratory performance of resting and swimming sockeye salmon (Oncorhynchus nerka). J. Exp. Biol. 2008, 211, 3915-3926. [CrossRef] [PubMed]

33. Karakach, T.K.; Huenupi, E.C.; Soo, E.C.; Walter, J.A.; Afonso, L.O.B. H-1-NMR and mass spectrometric characterization of the metabolic response of juvenile Atlantic salmon (Salmo salar) to long-term handling stress. Metabolomics 2009, 5, 123-137. [CrossRef]

34. Brandao, F.; Cappello, T.; Raimundo, J.; Santos, M.A.; Maisano, M.; Mauceri, A.; Pacheco, M.; Pereira, P. Unravelling the mechanisms of mercury hepatotoxicity in wild fish (Liza aurata) through a triad approach: Bioaccumulation, metabolomic profiles and oxidative stress. Metallomics 2015, 7, 1352-1363. [CrossRef] [PubMed]

35. Bowden, T.J. Modulation of the immune system of fish by their environment. Fish Shellfish Immun. 2008, 25, 373-383. [CrossRef] [PubMed]

36. Ratriyanto, A.; Mosenthin, R.; Bauer, E.; Eklund, M. Metabolic, Osmoregulatory and Nutritional Functions of Betaine in Monogastric Animals. Asian Australas. J. Anim. Sci. 2009, 22, 1461-1476. [CrossRef]

37. Kalujnaia, S.; Cramb, G. Regulation of Expression of the Myo-inositol Monophosphatase 1 Gene in Osmoregulatory Tissues of the European Eel Anguilla anguilla after Seawater Acclimation. Ann. N. Y. Acad. Sci. 2009, 1163, 433-436. [CrossRef]

38. Kultz, D. Physiological mechanisms used by fish to cope with salinity stress. J. Exp. Biol. 2015, 218, 1907-1914. [CrossRef]

39. Aragao, C.; Corte-Real, J.; Costas, B.; Dinis, M.T.; Conceicao, L.E.C. Stress response and changes in amino acid requirements in Senegalese sole (Solea senegalensis Kaup 1858). Amino Acids 2008, 34, 143-148. [CrossRef]

40. Petitjean, Q.; Jean, S.; Gandar, A.; Cote, J.; Laffaille, P.; Jacquin, L. Stress responses in fish: From molecular to evolutionary processes. Sci. Total Environ. 2019, 684, 371-380. [CrossRef]

41. Nakano, T.; Kameda, M.; Shoji, Y.; Hayashi, S.; Yamaguchi, T.; Sato, M. Effect of severe environmental thermal stress on redox state in salmon. Redox Biol. 2014, 2, 772-776. [CrossRef] [PubMed] 
42. Vinagre, C.; Madeira, D.; Narciso, L.; Cabral, H.N.; Diniz, M. Effect of temperature on oxidative stress in fish: Lipid peroxidation and catalase activity in the muscle of juvenile seabass, Dicentrarchus labrax. Ecol. Indic. 2012, 23, 274-279. [CrossRef]

43. Bonga, S.E.W. The stress response in fish. Physiol. Rev. 1997, 77, 591-625. [CrossRef] [PubMed]

44. Li, P.; Mai, K.S.; Trushenski, J.; Wu, G.Y. New developments in fish amino acid nutrition: Towards functional and environmentally oriented aquafeeds. Amino Acids 2009, 37, 43-53. [CrossRef] [PubMed]

45. Dani, V.D.; Lankadurai, B.P.; Nagato, E.G.; Simpson, A.J.; Simpson, M.J. Comparison of metabolomic responses of earthworms to sub-lethal imidacloprid exposure in contact and soil tests. Environ. Sci. Pollut. Res. 2019, 26, 18846-18855. [CrossRef] [PubMed]

46. Enrique Padilla-Guerrero, I.; Barelli, L.; Angelica Gonzalez-Hernandez, G.; Carlos Torres-Guzman, J.; Bidochka, M.J. Flexible metabolism in Metarhizium anisopliae and Beauveria bassiana: Role of the glyoxylate cycle during insect pathogenesis. Microbiology 2011, 157, 199-208. [CrossRef] [PubMed]

47. Somero, G.N.; Doyle, D. Temperature and rates of protein degradation in the fish Gillichthys mirabilis. Comp. Biochem. Physiol. Part B Comp. Biochem. 1973, 46, 463-474. [CrossRef]

48. Ip, A.Y.; Chew, S.F. Ammonia production, excretion, toxicity, and defense in fish: A review. Front. Physiol. 2010, 1. [CrossRef]

49. Andersen, S.M.; Waagbø, R.; Espe, M. Functional amino acids in fish health and welfare. Front. Biosci. 2016, 8, 143-169.

50. Lin, C.Y.; Wu, H.F.; Tjeerdema, R.S.; Viant, M.R. Evaluation of metabolite extraction strategies from tissue samples using NMR metabolomics. Metabolomics 2007, 3, 55-67. [CrossRef]

(C) 2019 by the authors. Licensee MDPI, Basel, Switzerland. This article is an open access article distributed under the terms and conditions of the Creative Commons Attribution (CC BY) license (http://creativecommons.org/licenses/by/4.0/). 